\title{
THE NORMAL DUAL CONGRUENCES AND THE DUAL BIANCHI LATTICE
}

\author{
ADAM DOLIWA \\ Wydzial Matematyki i Informatyki, Uniwersytet Warmińsko-Mazurski w Olsztynie, \\ ul. Żolnierska 14A, 10-561 Olsztyn, Poland \\ e-mail:doliwa@matman.uwm.edu.pl
}

(Received 12 December, 2003; accepted 30 April, 2004)

\begin{abstract}
The main goal of the paper is to find the discrete analogue of the Bianchi system in spaces of arbitrary dimension together with its geometric interpretation. We show that the proper geometric framework for such generalization is the language of dual quadrilateral lattices and of dual congruences. After introducing the notion of the dual Koenigs lattice in a projective space of arbitrary dimension, we define the discrete dual congruences and we present, as an important example, the normal dual discrete congruences. Finally, we introduce the dual Bianchi lattice as a dual Koenigs lattice allowing for a conjugate normal dual congruence, and we find its characterization in terms of a system of integrable difference equations.
\end{abstract}

2000 Mathematics Subject Classification. 37K25, 39A10, 52C99.

1. Introduction. In paper [2] Bianchi studied properties of the Moutard equation [26]

$$
\frac{\partial^{2} \overrightarrow{\boldsymbol{N}}}{\partial u \partial v}=f \overrightarrow{\boldsymbol{N}}
$$

subject to the quadratic reduction

$$
\frac{\partial^{2} \overrightarrow{\boldsymbol{N}}}{\partial u \partial v}=f \overrightarrow{\boldsymbol{N}}, \quad \overrightarrow{\boldsymbol{N}} \cdot \overrightarrow{\boldsymbol{N}}=U+V
$$

here $f$ is a given function of two variables $u$ and $v$, while $U$ and $V$ are functions of single variables $u$ and $v$, respectively. The unknown function $\vec{N}$ takes values in the space $\mathbb{R}^{M}$ equipped with the scalar product ".".

In the basic case of $M=3$ the above system is known as the Bianchi system and is related with the Ernst reduction of Einstein's equation (see the relevant literature in [27], where such correpondence was also generalized to the case of $M>3$ ). In classical differential geometry the Bianchi system for $M=3$ appears in the contexts of:

(i) conjugate nets permanent in deformation [22];

(ii) conjugate nets with equal tangential invariants allowing for harmonic normal congruence [23];

(iii) asymptotic nets allowing for the Weingarten transformations preserving the Gauss curvature [3]. 
In the interpretations (i) and (ii), which in fact describe the same geometric object from different points of view, the variables $u, v$ are conjugate parameters. In the case (iii) the variables $u, v$ are the asymptotic coordinates on a hyperbolic surface.

The Bianchi system is, due to its physical importance, one of the most interesting soliton systems having the distinguished geometric meaning. During recent years, integrable discrete analogues of many geometrically meaningful integrable systems have been found (see, for example, $[6,18,19,20,21,24,28]$ or a recent review [11]).

The discrete integrable analogue of the Bianchi system for $M=3$ has been obtained in $[\mathbf{1 7}, \mathbf{3 0}]$ as a reduction of the discrete Moutard equation [31] which describes asymptotic lattices $[\mathbf{1 0}, \mathbf{2 8}]$, i.e. via a discrete version of the interpretation (iii). It turns out that the same equation was obtained in the earlier work [32], for arbitrary $M$, in a different geometric context of isothermic lattices [5].

An alternative approach, based on the interpretation (ii), to the integrable discretization of the Bianchi system (again for $M=3$ ) was presented in [16]. Suprisingly, one obtains in this way not the system derived in $[\mathbf{1 7}, \mathbf{3 0}]$, but an integrable reduction of the Koenigs lattice equation [13, 29]. There exists, however, a simple relation between both discrete versions of the Bianchi system. We remark also that the interpretation (i), has recently been discretized [33].

The algebraic transition to $M>3$ of the discrete Bianchi system obtained in [16] does not need special efforts. The main goal of the paper is to find the proper geometric framework for such a generalization. We define therefore, in addition to the recently introduced notion of the dual quadrilateral lattice [20], the notion of the discrete dual congruence, thus making the theory of quadrilateral lattices [19] and their transformations [21] perfectly dual (in the sense of the standard duality in projective spaces).

The layout of the paper is as follows. We first recall the definition of the dual quadrilateral lattice and we introduce the dual Koenigs lattice in a projective space of arbitrary dimension. In Section 3 we define the discrete dual congruences and we study their basic properties. The next Section 4 is devoted to an important class of such congruences, the normal dual discrete congruences. Then, in Section 5 we introduce the dual Bianchi lattice as a dual Koenigs lattice allowing for a conjugate normal dual congruence. Finally, we characterize the dual Bianchi lattices in terms of solutions of the discrete Bianchi system and we provide the Darboux-type transformation for the system.

2. The dual Koenigs lattice. The dual quadrilateral lattices, introduced in [20], are lattices of planar quadrilaterals in the dual space $\left(\mathbb{P}^{M}\right)^{*}$.

Definition 1. A map $x^{*}: \mathbb{Z}^{2} \rightarrow\left(\mathbb{P}^{M}\right)^{*}, M>2$, from a two dimensional integer lattice into the space of hyperplanes in $M$-dimensional projective space $\mathbb{P}^{M}$ is called a dual quadrilateral lattice if the intersection of any four neighbouring hyperplanes $x^{*}$, $x_{(1)}^{*}, x_{(2)}^{*}$ and $x_{(12)}^{*}$ is a projective subspace of co-dimension three.

By subscripts in brackets we denote shifts in the corresponding discrete variables, for example $f_{( \pm 1)}\left(m_{1}, m_{2}\right)=f\left(m_{1} \pm 1, m_{2}\right)$.

REMARK. We will consider here only non-degenerate dual quadrilateral lattices for which no three of the four neighbouring hyperplanes have intersection of co-dimension two. 
REMARK. The case of $M=3$ is special, because any quadrilateral lattice $x: \mathbb{Z}^{2} \rightarrow$ $\mathbb{P}^{3}$ gives rise to a dual quadrilateral lattice $x^{*}: \mathbb{Z}^{2} \rightarrow\left(\mathbb{P}^{3}\right)^{*}$, where $x^{*}$ is the plane passing through the points $x, x_{(1)}$ and $x_{(2)}$.

The homogeneous coordinates $\boldsymbol{x}^{*}: \mathbb{Z}^{2} \rightarrow \mathbb{R}^{M+1} \backslash\{0\}$ of the dual quadrilateral lattices are subjected to the discrete Laplace equation

$$
x_{(12)}^{*}=A_{(1)}^{*} x_{(1)}^{*}+B_{(2)}^{*} x_{(2)}^{*}+C^{*} x^{*} .
$$

Denote by $L_{i}^{*}$ the subspace of co-dimension two (a dual line) being the intersection of $x^{*}$ and $x_{(i)}^{*}, i=1,2$. The hyperplanes containing $L_{i}^{*}$ (such a pencil of hyperplanes is often identified with $L_{i}^{*}$ ) have coordinates $\lambda \boldsymbol{x}^{*}+\mu \boldsymbol{x}_{(i)}^{*}$. The hyperplanes $x_{-1}^{*}$ with homogeneous coordinates

$$
\boldsymbol{x}_{-1}^{*}=\boldsymbol{x}_{(1)}^{*}-B^{*} \boldsymbol{x}^{*}=A_{(1-2)}^{*} \boldsymbol{x}_{(1-2)}^{*}+C_{(-2)}^{*} \boldsymbol{x}_{(-2)}^{*},
$$

are contained in the corresponding pencils $L_{1}^{*}$ and $L_{1(-2)}^{*}$. Similarly, the hyperplanes $x_{1}^{*}$ with homogeneous coordinates

$$
\boldsymbol{x}_{1}^{*}=\boldsymbol{x}_{(2)}^{*}-A^{*} \boldsymbol{x}^{*}=B_{(-12)}^{*} \boldsymbol{x}_{(-12)}^{*}+C_{(-1)}^{*} \boldsymbol{x}_{(-1)}^{*},
$$

are contained in the pencils $L_{2}^{*}$ and $L_{2(-1)}^{*}$.

REMARK. Above we defined the Laplace transforms [8] of dual quadrilateral lattices.

A distinguished example of the integrable dual lattice is the dual Koenigs lattice (for definition of the Koenigs lattice see [13]), introduced in an equivalent form in the special case $M=3$ in [16].

DeFINITION 2. A dual quadrilateral lattice $x^{*}: \mathbb{Z}^{2} \rightarrow\left(\mathbb{P}^{M}\right)^{*}$ is called a dual Koenigs lattice if the three pencils: (i) containing the hyperplanes $x_{1}^{*}$ and $x_{-1(22)}^{*}$, (ii) containing the hyperplanes $x_{-1}^{*}$ and $x_{1(11)}^{*}$, (iii) containing the hyperplanes $x^{*}$ and $x_{(12)}^{*}$, have a hyperplane in common.

Remark. For generic dual quadrilateral lattice these three pencils contain the projective subspace $x^{*} \cap x_{(1)}^{*} \cap x_{(2)}^{*}$ of co-dimension three.

To characterize algebraically the dual Koenigs lattices we present the corresponding reduction of the general discrete Laplace equation for dual quadrilateral lattices.

THEOREM 1. A dual quadrilateral lattice $x^{*}: \mathbb{Z}^{2} \rightarrow\left(\mathbb{P}^{M}\right)^{*}$ is a dual Koenigs lattice if and only if its Laplace equation can be gauged into the form

$$
x_{(12)}^{*}+x^{*}=F_{(1)}^{*} x_{(1)}^{*}+F_{(2)}^{*} x_{(2)}^{*} .
$$

Proof. We will follow the reasoning of [16]. There exists a common hyperplane of the three pencils if and only if the linear system

$$
\lambda \boldsymbol{x}_{1}^{*}+\mu \boldsymbol{x}_{-1(22)}^{*}=\sigma \boldsymbol{x}_{-1}^{*}+\rho \boldsymbol{x}_{1(11)}^{*}=\chi \boldsymbol{x}^{*}+\nu \boldsymbol{x}_{(12)}^{*}
$$

has a non-trivial solution $\lambda, \mu, \sigma, \rho, \chi, \nu$. Using equations (2)-(3) one can rewrite the linear equations (5) in the form

$$
\begin{aligned}
& \lambda\left(x_{(2)}^{*}-A^{*} x^{*}\right)+\mu\left(A_{(12)}^{*} x_{(12)}^{*}+C_{(2)}^{*} x_{(2)}^{*}\right) \\
& \quad=\sigma\left(x_{(1)}^{*}-B^{*} x^{*}\right)+\rho\left(B_{(12)}^{*} x_{(12)}^{*}+C_{(1)}^{*} x_{(1)}^{*}\right)=\chi x^{*}+v x_{(12)}^{*} .
\end{aligned}
$$


With the help of the Laplace equation (1) one concludes that such a solution exists if and only if the coefficients of the Laplace equation are restricted by the following equation

$$
A^{*} C_{(2)}^{*} B_{(12)}^{*}=B^{*} C_{(1)}^{*} A_{(12)}^{*}
$$

This restriction on the coefficients of the Laplace equation (1) implies [13] existence of the gauge function $\rho$ defined by

$$
\rho_{(12)}=-C^{*} \rho, \quad \rho_{(1)} A^{*}=\rho_{(2)} B^{*} .
$$

After the gauge transformation $\boldsymbol{x}^{*} \mapsto \boldsymbol{x}^{*} / \rho$, the new coordinates of the dual lattice satisfy the Laplace equation of the form (4) with the potential

$$
F^{*}=\frac{A^{*} \rho}{\rho_{(2)}}=\frac{B^{*} \rho}{\rho_{(1)}} .
$$

In [13], the reduction of the fundamental transformation acting within the class of lattices whose homogeneous coordinates satisfy equation (4) was also found. The geometric content of the following result will be presented in the next section.

Proposition 2. Given the dual Koenigs lattice $x^{*}: \mathbb{Z}^{2} \rightarrow\left(\mathbb{P}^{M}\right)^{*}$ with homogeneous coordinates $\boldsymbol{x}^{*}$ satisfying equation (4) and given a scalar solution $\theta$ of its adjoint equation (the Moutard equation)

$$
\theta_{(12)}+\theta=F^{*}\left(\theta_{(1)}+\theta_{(2)}\right)
$$

define the functions

$$
\phi=\theta_{(1)}+\theta_{(2)}, \quad \phi^{\prime}=\frac{1}{\theta_{(1)}}+\frac{1}{\theta_{(2)}} .
$$

Then the solution $\boldsymbol{x}^{* \prime}$ of the linear system

$$
\begin{aligned}
& \Delta_{1}\left(\frac{\boldsymbol{x}^{* \prime}}{\phi^{\prime}}\right)=\left(\theta \theta_{(2)}\right)_{(1)} \Delta_{1}\left(\frac{\boldsymbol{x}^{*}}{\boldsymbol{\phi}}\right), \\
& \Delta_{2}\left(\frac{\boldsymbol{x}^{* \prime}}{\phi^{\prime}}\right)=-\left(\theta \theta_{(1)}\right)_{(2)} \Delta_{2}\left(\frac{\boldsymbol{x}^{*}}{\boldsymbol{\phi}}\right),
\end{aligned}
$$

satisfies equation (4) with

$$
F^{* \prime}=F^{*} \frac{\theta_{(1)} \theta_{(2)}}{\theta \theta_{(12)}},
$$

and defines homogeneous coordinates of the new dual Koenigs lattice.

REMARK. The Koenigs lattice equation (4) was introduced in [29] in the context of Laplace sequences of quadrilateral lattices. 
3. Dual congruences. In this section we introduce the dual version of the second basic object of the discrete integrable geometry, the notion of a discrete dual congruence. In what follows by $\mathbb{G}(K+1, M+1)$ we denote the set of $(K+1)$ dimensional linear subspaces of the $(M+1)$-dimensional vector space $\mathbb{R}^{M+1}$, i.e. the space of $K$-dimensional projective subspaces of the $M$-dimensional projective space $\mathbb{P}^{M}$.

Definition 3. A map $L^{*}: \mathbb{Z}^{2} \rightarrow \mathbb{G}(M-1, M+1), M>2$, is called a dual congruence if any two neighbouring subspaces are contained in a subspace of codimension one. The lattices of common hyperplanes $y_{i}^{*}: \mathbb{Z}^{2} \rightarrow\left(\mathbb{P}^{M}\right)^{*}, i=1,2$, of $L^{*}$ and $L_{(-i)}^{*}$ are called focal hyperplane lattices (or dual focal lattices) of the dual congruence.

REMARK. We will consider here only non-degenerate dual congruences for which any subspace of the congruence cannot have common hyperplanes with other subspaces different from its nearests neighbours.

COROLlary 3. The Grassmann space $\mathbb{G}(M-1, M+1)$ is the natural dual of the space $\mathbb{G}(2, M+1)$ of lines in $\mathbb{P}^{M}$. Moreover, the dual version of two intersecting lines are two projective subspaces of co-dimension two contained in a hyperplane. Therefore, all known results of the theory of discrete congruences [21] have the natural dual counterpart.

In particular, given the dual quadrilateral lattice $x^{*}: \mathbb{Z}^{2} \rightarrow\left(\mathbb{P}^{M}\right)^{*}$ then the maps $L_{i}^{*}: \mathbb{Z}^{2} \rightarrow \mathbb{G}(M-1, M+1), i=1,2$, defined by $L_{i}^{*}=x^{*} \cap x_{(i)}^{*}$ and considered in the previous section, are dual congruences. They are the dual versions of the tangent congruences $L_{i}: \mathbb{Z}^{2} \rightarrow \mathbb{G}(2, M+1)$ of a quadrilateral lattice $x: \mathbb{Z}^{2} \rightarrow \mathbb{P}^{M}$, which are given by lines $L_{i}$ passing through $x$ and $x_{(i)}$.

REMARK. In the case $M=3$ the notion of line is self-dual, and any congruence is simultaneously a dual congruence as well. However, if $x: \mathbb{Z}^{2} \rightarrow \mathbb{P}^{3}$ is a quadrilateral lattice and $x^{*}: \mathbb{Z}^{2} \rightarrow\left(\mathbb{P}^{3}\right)^{*}$ is the dual quadrilateral lattice of its tangent planes, then $L_{1}^{*}=L_{2(1)}$ and $L_{2}^{*}=L_{1(2)}$.

Analogously, we have in the case $M=3$ the following double interpretation of "focal planes" of the congruence, which had to be taken into account in [16] (see Figure 1).

Corollary 4. Let $L=L^{*}: \mathbb{Z}^{2} \rightarrow \mathbb{G}(2,4)$ be a congruence in $\mathbb{P}^{3}$ and let $y_{i}^{*}: \mathbb{Z}^{2} \rightarrow$ $\left(\mathbb{P}^{3}\right)^{*}, i=1,2$, denote its dual focal lattices. If by $\tilde{y}_{i}^{*}: \mathbb{Z}^{2} \rightarrow\left(\mathbb{P}^{3}\right)^{*}$ we denote the dual lattices of tangent planes of the focal (point) lattices $\tilde{y}_{i}: \mathbb{Z}^{2} \rightarrow \mathbb{P}^{3}, i=1,2$, of the congruence, then $\tilde{y}_{1}^{*}=y_{2(2)}^{*}$ and $\tilde{y}_{2}^{*}=y_{1(1)}^{*}$.

Proposition 5. If $L^{*}: \mathbb{Z}^{2} \rightarrow \mathbb{G}(M-1, M+1)$ is a dual congruence then its focal hyperplane lattices $y_{i}^{*}: \mathbb{Z}^{2} \rightarrow\left(\mathbb{P}^{M}\right)^{*}, i=1,2$, are quadrilateral hyperplane lattices.

Proof. We will demonstrate the Proposition for the first focal dual lattice. Denote by $\boldsymbol{y}_{1}^{*}: \mathbb{Z}^{2} \rightarrow \mathbb{R}^{M+1} \backslash\{0\}$, its homogeneous representants. Because both hyperplanes $y_{1}^{*}$ and $y_{1(1)}^{*}$ contain the subspace $L^{*}$ then any other hyperplane of this pencil has homogeneous coordinates of the form

$$
\alpha \boldsymbol{y}_{1}^{*}+\beta \boldsymbol{y}_{1(1)}^{*}
$$




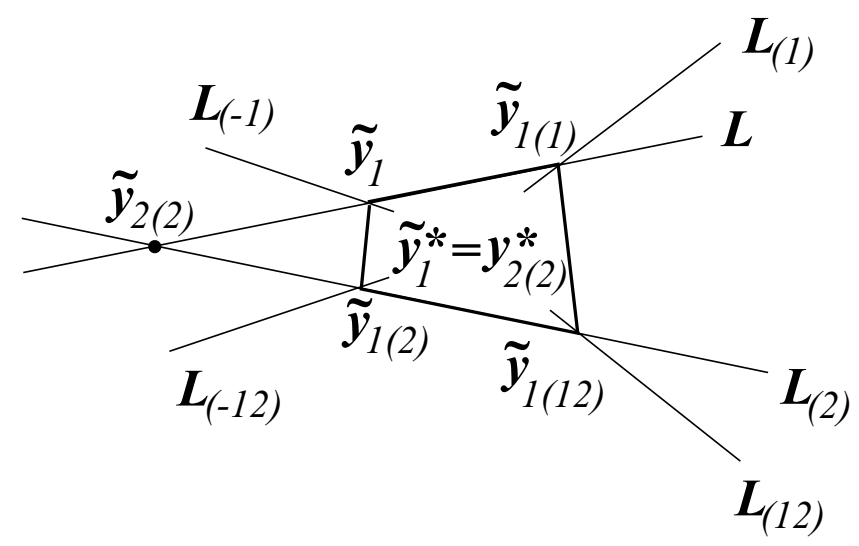

Figure 1. The focal planes of a congruence in $\mathbb{P}^{3}$

Similarly, the homogeneous coordinates of any hyperplane passing through $L_{(2)}^{*}$ are a linear combination of $\boldsymbol{y}_{1(2)}^{*}$ and $\boldsymbol{y}_{1(12)}^{*}$

$$
\gamma \boldsymbol{y}_{1(2)}^{*}+\delta \boldsymbol{y}_{1(12)}^{*} \text {. }
$$

Because the hyperplane $y_{2(2)}^{*}$ is contained in both pencils then for some non-zero $\lambda$ and not all vanishing $\alpha, \beta, \gamma, \delta$

$$
\lambda\left(\alpha \boldsymbol{y}_{1}^{*}+\beta \boldsymbol{y}_{1(1)}^{*}\right)=\gamma \boldsymbol{y}_{1(2)}^{*}+\delta \boldsymbol{y}_{1(12)}^{*} .
$$

COROLlary 6. Notice that $\delta=0$ would imply that $L^{*}$ is contained in $y_{1(2)}^{*}$, which violates the assumption of genericity of the dual congruence.

REMARK. The notion of the dual congruences can be naturally extended to $N$-parameter families of dual lines. The proof that the focal hyperplane lattices are quadrilateral, however then needs additional genericity assumptions (see [21] where the analogous problem for congruences of lines was treated in detail).

The dual congruences play the same role in the theory of transformations of quadrilateral dual lattices as the congruences of lines in the theory of transformations of quadrilateral lattices.

Definition 4. A hyperplane quadrilateral lattice $x^{*}: \mathbb{Z}^{2} \rightarrow\left(\mathbb{P}^{M}\right)^{*}$ and a dual congruence $L^{*}: \mathbb{Z}^{2} \rightarrow \mathbb{G}(M-1, M+1)$ are said to be conjugate to each other if the subspaces $L^{*}$ of the dual congruence are contained in the corresponding hyperplanes $x^{*}$ of the lattice.

REMARK. For $M=3$ when the notion of a congruence is self-dual, if $x: \mathbb{Z}^{2} \rightarrow \mathbb{P}^{3}$ is a quadrilateral lattice and $x^{*}: \mathbb{Z}^{2} \rightarrow\left(\mathbb{P}^{3}\right)^{*}$ is the dual quadrilateral lattice of its tangent planes, then a (dual) congruence $L^{*}=L: \mathbb{Z}^{2} \rightarrow \mathbb{G}(2,4)$ conjugate to $x^{*}$ is called harmonic to $x$.

DEFINITION 5. Two quadrilateral dual lattices are related by the dual fundamental transformation if they are conjugated to the same dual congruence. 
The algebraic results of the theory of transformations of the quadrilateral lattices can be interpreted in the dual space. In particular, we present here the dual geometric content of the formulas, originally obtained for the Koenigs lattices in [13]. The restriction of the dual fundamental transformation to the class of the dual Koenigs lattices is algebraically formulated in Theorem 2.

Corollary 7. Let $\boldsymbol{x}^{*}: \mathbb{Z}^{2} \rightarrow \mathbb{R}^{M+1} \backslash\{0\}$ and $\boldsymbol{x}^{* \prime}: \mathbb{Z}^{2} \rightarrow \mathbb{R}^{M+1} \backslash\{0\}$ be the homogeneous representants of two dual Koenigs lattices related by the dual Koenigs transformation described in Theorem 2. The homogeneous representants of the dual focal lattices of the dual congruence of that transformation read

$$
\boldsymbol{y}_{1}^{*}=\theta \theta_{(2)} \frac{\boldsymbol{x}^{*}}{\phi}-\frac{\boldsymbol{x}^{* \prime}}{\phi^{\prime}}, \quad \boldsymbol{y}_{2}^{*}=-\theta \theta_{(1)} \frac{\boldsymbol{x}^{*}}{\phi}-\frac{\boldsymbol{x}^{* \prime}}{\phi^{\prime}} .
$$

Moreover, they can be found from equations

$$
\begin{aligned}
\boldsymbol{y}_{1}^{*}-\boldsymbol{y}_{2}^{*} & =\theta \boldsymbol{x}^{*}, \\
\Delta_{1} \boldsymbol{y}_{2}^{*} & =\left(F^{*} \boldsymbol{x}^{*}-\boldsymbol{x}_{(1)}^{*}\right) \theta_{(1)}, \\
\Delta_{2} \boldsymbol{y}_{1}^{*} & =\left(\boldsymbol{x}_{(2)}^{*}-F^{*} \boldsymbol{x}^{*}\right) \theta_{(2)} .
\end{aligned}
$$

Given a dual congruence $L^{*}: \mathbb{Z}^{2} \rightarrow \mathbb{G}(M-1, M+1)$ with dual focal lattices $y_{i}^{*}$ : $\mathbb{Z}^{2} \rightarrow\left(\mathbb{P}^{M}\right)^{*}, i=1,2$. In the pencil of hyperplanes containing $L^{*}$ define the projective involution $\mathfrak{i}_{L^{*}}$ with two pairs of homologous hyperplanes $y_{i(i)}^{*}=\mathfrak{i}_{L^{*}}\left(y_{i}^{*}\right), i=1,2$. The dual Koenigs lattices can be geometrically selected from generic quadrilateral lattices by the following property, being the dual version of Corollary 15 of [13].

Proposition 8. A dual quadrilateral lattice $x^{*}: \mathbb{Z}^{2} \rightarrow\left(\mathbb{P}^{M}\right)^{*}$ is a dual Koenigs lattice if and only if, for arbitrary non-degenerate dual congruence $L^{*}: \mathbb{Z}^{2} \rightarrow \mathbb{G}(M-1, M+1)$ conjugate to the lattice, the dual lattice $x^{* \prime}: \mathbb{Z}^{2} \rightarrow\left(\mathbb{P}^{M}\right)^{*}$, defined by $x^{* \prime}=\mathfrak{i}_{L^{*}}(x)$ is quadrilateral as well.

COROLlary 9. In the notation of Theorem 2 and Corollary 7 the homogeneous coordinates of the fixed hyperplanes $y_{ \pm}^{*}$ of the involutions $\mathfrak{i}_{L^{*}}$ read

$$
\boldsymbol{y}_{ \pm}^{*}= \pm \sqrt{\theta \theta_{(12)}} \boldsymbol{x}^{*}+\sqrt{\theta_{(1)} \theta_{(2)}} \boldsymbol{x}^{* \prime} .
$$

4. The normal dual congruences. In this section we define the normal dual congruences. The normal congruences in the (self-dual) case $M=3$ were defined in [16] following the example (see [12]) of normal congruences of circular lattices in three dimensional Euclidean space $\mathbb{E}^{3}$. The circular lattice is a quadrilateral lattice whose quadrilaterals can be inscribed in circles, and its properties have been studied in detail in $[\mathbf{1}, \mathbf{4}, \mathbf{7}, \mathbf{9}, \mathbf{1 4}, \mathbf{1 5}, \mathbf{2 4}, \mathbf{2 5}]$. In [12] it was shown that the normals $v$ to circles of the circular lattice $x: \mathbb{Z}^{2} \rightarrow \mathbb{E}^{3}$ passing through the centers of circles (see Figure 2) form a congruence. In [16] such congruences were characterized without any reference to a circular lattice, which was then used to define normal congruences in $\mathbb{E}^{3}$ as follows (by $\mathbb{G}_{A}(K+1, M+1)$ we denote the set of $K$-dimensional (affine) subspaces of the $M$-dimensional Euclidean space $\left.\mathbb{E}^{M}\right)$.

Definition 6. A congruence $v: \mathbb{Z}^{2} \rightarrow \mathbb{G}_{A}(2,4)$ with focal lattices $\tilde{y}_{i}: \mathbb{Z}^{2} \rightarrow \mathbb{E}^{3}$, $i=1,2$, is a normal congruence if the pair of orthogonal planes bisecting the angles between the planes $\tilde{y}_{1(-2)}^{*}$ and $\tilde{y}_{1}^{*}$ coincides with those bisecting $\tilde{y}_{2(-1)}^{*}$ and $\tilde{y}_{2}^{*}$. 


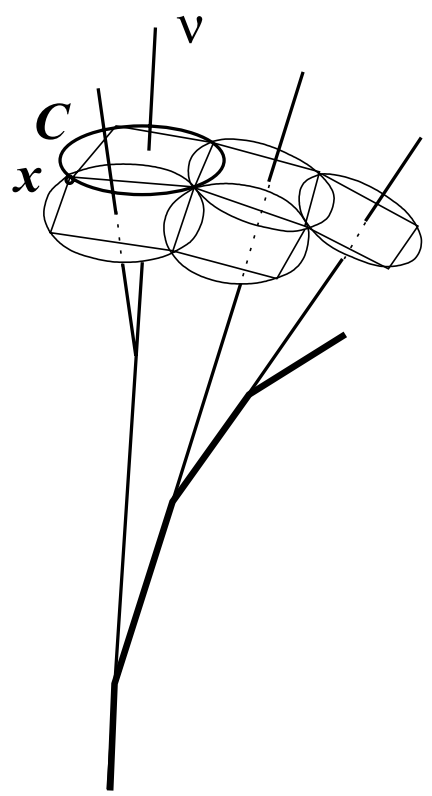

Figure 2. The normal congruence of a circular lattice in $\mathbb{E}^{3}$

Taking into account self-duality of lines in $\mathbb{P}^{3}$ and Corollary 4 , one can extend the above definition to $M>3$.

Definition 7. A dual congruence $v^{*}: \mathbb{Z}^{2} \rightarrow \mathbb{G}_{A}(M-1, M+1)$ with dual focal lattices $y_{i}^{*}: \mathbb{Z}^{2} \rightarrow \mathbb{E}^{M}, i=1,2$, is a normal dual congruence if the pair of orthogonal hyperplanes bisecting the angles between the hyperplanes $y_{2}^{*}$ and $y_{2(2)}^{*}$ coincides with those bisecting $y_{1}^{*}$ and $y_{1(1)}^{*}$.

COROLlary 10. Given a circular lattice $x: \mathbb{Z}^{2} \rightarrow \mathbb{E}^{M}$, then the unique subspaces $v^{*}$ of co-dimension two, orthogonal to the planes of circles of the lattice and containing the centers of the circles, form a normal dual congruence.

Denote by $y_{ \pm}^{*}$ the pair of hyperplanes containing the subspace $v^{*}$ of a normal dual congruence $v^{*}: \mathbb{Z}^{2} \rightarrow \mathbb{G}_{A}(M-1, M+1)$ and bisecting the pair of hyperplanes $y_{2}^{*}$ and $y_{2(2)}^{*}\left(\right.$ or $y_{1}^{*}$ and $\left.y_{1(1)}^{*}\right)$. Denote by $\mathfrak{r}_{v^{*}}$ the unique reflection in the pencil of hyperplanes containing $v^{*}$ such that $y_{2}^{*}$ and $y_{2(2)}^{*}$ are homologous, i.e. $\mathfrak{r}_{v^{*}}\left(y_{2}^{*}\right)=y_{2(2)}^{*}$. Then the hyperplanes $y_{ \pm}^{*}$ are the fixed hyperplanes of the reflection and also $y_{1}^{*}$ is homologous to $y_{1(1)}^{*}$.

Recall that, within all projective involutions in a pencil of hyperplanes in $\mathbb{E}^{M}$, the reflections are characterized by the property that the fixed hyperplanes of the involution are orthogonal. This gives the following characterization of the normal dual congruences.

Proposition 11. Given a dual congruence $L^{*}: \mathbb{Z}^{2} \rightarrow \mathbb{G}_{A}(M-1, M+1)$ with dual focal lattices $y_{i}^{*}: \mathbb{Z}^{2} \rightarrow \mathbb{E}^{M}, i=1,2$. In the pencils of hyperplanes containing the dual lines $L^{*}$ of the dual congruence consider the projective involutions $\mathfrak{i}_{L^{*}}$, with two pairs of homologous hyperplanes $y_{i(i)}^{*}=\mathfrak{i}_{L^{*}}\left(y_{i}^{*}\right)$. The congruence is normal if and only if the fixed hyperplanes of the involutions are orthogonal. 
5. The dual quadrilateral Bianchi lattice. The following definition is a generalization, to the dimension $M \geq 3$ of the ambient space, of the definition of quadrilateral Bianchi lattice given in [16] for $M=3$.

DEFINITION 8. The dual quadrilateral Bianchi lattice is a dual Koenigs lattice in $\mathbb{E}^{M}$ allowing for a conjugate normal dual congruence.

Given a hyperplane $w^{*}$ in $\mathbb{E}^{M}$ then its homogeneous coordinates $\boldsymbol{w}^{*}=\left(\overrightarrow{\boldsymbol{\omega}}, \omega^{M+1}\right)$ are given up to a multiplication by a non-zero factor. Then $\vec{\omega}$ is a vector normal (not necessarily unit) to the hyperplane and the point $u \in \mathbb{E}^{M}$ with coordinates $\overrightarrow{\boldsymbol{u}} \in \mathbb{R}^{M}$ belongs to the plane $w$ if $\overrightarrow{\boldsymbol{\omega}} \cdot \overrightarrow{\boldsymbol{u}}+\omega^{M+1}=0$.

Corollary 12. A dual quadrilateral lattice $x^{*}: \mathbb{Z}^{2} \rightarrow \mathbb{G}_{A}(M, M+1)$ is a dual Koenigs lattice if and only if the field of normal vectors $\overrightarrow{\boldsymbol{n}}: \mathbb{Z}^{2} \rightarrow \mathbb{E}^{M}$ to hyperplanes of the lattice can be chosen in such a way that it satisfies the discrete Koenigs equation (4).

Let us formulate the central result of this section. The idea of its proof follows the corresponding geometric reasoning of [16].

THEOREM 13. The dual lattice $x^{*}: \mathbb{Z}^{2} \rightarrow \mathbb{G}_{A}(M, M+1)$ is a Bianchi lattice if and only if its normal vector $\overrightarrow{\boldsymbol{n}}: \mathbb{Z}^{2} \rightarrow \mathbb{E}^{M}$ can be chosen in such a way that it satisfies the discrete Koenigs equation (4) with the potential $F^{*}: \mathbb{Z}^{2} \rightarrow \mathbb{R}$ subjected to the condition

$$
\Delta_{1} \Delta_{2}\left(\overrightarrow{\boldsymbol{n}} \cdot \overrightarrow{\boldsymbol{n}} F^{*}\right)=0 .
$$

Proof. $\Rightarrow$ Consider the dual Bianchi lattice $x^{*}$ and choose its homogeneous coordinates $\boldsymbol{x}^{*}=\left(\overrightarrow{\boldsymbol{n}}, x^{*(M+1)}\right)$ satisfying the discrete Koenigs equation (4). Let $L^{*}$ be a normal dual congruence conjugate to $x^{*}$, let $y_{i}^{*}, i=1,2$, denote the focal dual lattices of the congruence, then by Theorem 2, Corollary 7 and Proposition 8 there exists a solution $\theta$ of the discrete Moutard equation (6) and the homogeneous coordinates $\left(\overrightarrow{\boldsymbol{n}}_{i}, y_{i}^{*(M+1)}\right)$ of the focal lattices can be chosen such that they satisfy the system (12).

By $\mathfrak{i}_{L^{*}}$ denote the involutions in pencils of the dual congruence, defined by conditions $y_{i(i)}^{*}=\mathfrak{i}_{L^{*}}\left(y_{i}\right), i=1,2$. Then the corresponding dual Koenigs lattice $x^{* \prime}=$ $\mathfrak{i}_{L^{*}}\left(x^{*}\right)$, has the homogeneous coordinates $\left(\overrightarrow{\boldsymbol{n}}^{\prime}, x^{*(M+1)}\right)$ satisfying the discrete Koenigs equation (4) with the potential $F^{* \prime}$ given by equation (10). Notice that because $x^{* \prime}$ is conjugate to the normal dual congruence $L^{*}$, then $x^{* \prime}$ is a Bianchi lattice as well.

According to Corollary 9 the fixed hyperplanes $y_{ \pm}^{*}$ of the involution $\mathfrak{i}_{L^{*}}$ have the normal vectors given by

$$
\overrightarrow{\boldsymbol{n}}_{ \pm}= \pm \sqrt{\theta \theta_{(12)}} \overrightarrow{\boldsymbol{n}}+\sqrt{\theta_{(1)} \theta_{(2)}} \overrightarrow{\boldsymbol{n}}^{\prime}
$$

Because the dual $L^{*}$ congruence is normal then Proposition 11 implies that

$$
\overrightarrow{\boldsymbol{n}}_{+} \cdot \overrightarrow{\boldsymbol{n}}_{-}=0
$$

which, due to equations (15) and (10), gives

$$
\overrightarrow{\boldsymbol{n}} \cdot \overrightarrow{\boldsymbol{n}} F^{*}=\overrightarrow{\boldsymbol{n}}^{\prime} \cdot \overrightarrow{\boldsymbol{n}}^{\prime} F^{* \prime}
$$

Equations (7), (11) and (16) imply that

$$
\frac{\overrightarrow{\boldsymbol{n}}_{1} \cdot \overrightarrow{\boldsymbol{n}}_{1}}{\theta \theta_{(2)}}+\frac{\overrightarrow{\boldsymbol{n}}_{2} \cdot \overrightarrow{\boldsymbol{n}}_{2}}{\theta \theta_{(1)}}=\overrightarrow{\boldsymbol{n}} \cdot \overrightarrow{\boldsymbol{n}} F^{*} .
$$


Moreover, the same equations supplemented by the transformation formulas (8)-(9) give conditions

$$
\Delta_{1}\left(\frac{\overrightarrow{\boldsymbol{n}}_{1} \cdot \overrightarrow{\boldsymbol{n}}_{1}}{\theta \theta_{(2)}}\right)=0, \quad \Delta_{2}\left(\frac{\overrightarrow{\boldsymbol{n}}_{2} \cdot \overrightarrow{\boldsymbol{n}}_{2}}{\theta \theta_{(1)}}\right)=0,
$$

which together with equation (17) lead to the constraint (14).

$\Leftarrow$ By Corollary 12 the dual lattice $x^{*}$ is a dual Koenigs lattice. Denote by $\boldsymbol{x}^{*}=$ $\left(\vec{n}, x^{*(M+1)}\right)$ its homogeneous coordinates satisfying the discrete Koenigs equation (4). The constraint (14) implies that there exist functions $U_{1}$ and $U_{2}$ of the single variables $m_{1}$ and $m_{2}$, respectively, such that

$$
\overrightarrow{\boldsymbol{n}} \cdot \overrightarrow{\boldsymbol{n}} F^{*}=U_{1}+U_{2} .
$$

We will construct a one-parameter family of normal dual congruences conjugated to the lattice $x^{*}$. The parameter $\lambda$, present in the construction, will play the role of the spectral parameter of the soliton theory.

Consider the following system of equations

$$
\begin{aligned}
& \theta_{(1)}=\frac{(\theta \overrightarrow{\boldsymbol{n}}+\overrightarrow{\boldsymbol{\omega}}) \cdot(\theta \overrightarrow{\boldsymbol{n}}+\overrightarrow{\boldsymbol{\omega}})}{4 \theta\left(U_{1}+\lambda\right)}, \\
& \theta_{(2)}=\frac{(\theta \overrightarrow{\boldsymbol{n}}-\overrightarrow{\boldsymbol{\omega}}) \cdot(\theta \overrightarrow{\boldsymbol{n}}-\overrightarrow{\boldsymbol{\omega}})}{4 \theta\left(U_{2}-\lambda\right)},
\end{aligned}
$$

and

$$
\begin{aligned}
& \Delta_{1} \boldsymbol{w}^{*}=\theta_{(1)} \boldsymbol{x}_{(1)}^{*}-\left(2 F^{*} \theta_{(1)}-\theta\right) \boldsymbol{x}^{*}, \\
& \Delta_{2} \boldsymbol{w}^{*}=-\theta_{(2)} \boldsymbol{x}_{(2)}^{*}+\left(2 F^{*} \theta_{(2)}-\theta\right) \boldsymbol{x}^{*},
\end{aligned}
$$

where $\boldsymbol{w}=\left(\overrightarrow{\boldsymbol{\omega}}, \omega^{M+1}\right): \mathbb{Z}^{2} \rightarrow \mathbb{R}^{M+1}$ and $\theta: \mathbb{Z}^{2} \rightarrow \mathbb{R}$ are unknown fields. In proving compatibility of the system (19)-(22) it is important to notice the following consequences of equations (19)-(20) and of the constraint (14)

$$
\begin{aligned}
& \overrightarrow{\boldsymbol{\omega}} \cdot \overrightarrow{\boldsymbol{n}}=\theta_{(1)}\left(U_{1}+\lambda\right)-\theta_{(2)}\left(U_{2}-\lambda\right), \\
& \overrightarrow{\boldsymbol{\omega}} \cdot \overrightarrow{\boldsymbol{\omega}}=2\left(\left(U_{1}+\lambda\right) \theta \theta_{(1)}+\left(U_{2}-\lambda\right) \theta \theta_{(2)}\right)-\theta^{2} \frac{U_{1}+U_{2}}{F^{*}} .
\end{aligned}
$$

Moreover, in checking compatibility of the system one verifies that $\theta$ satisfies the discrete Moutard equation (6).

The dual lattices $y_{i}^{*}: \mathbb{Z}^{2} \rightarrow \mathbb{G}_{A}(M, M+1), i=1,2$, with the homogeneous coordinates $\boldsymbol{y}_{i}^{*}=\left(\overrightarrow{\boldsymbol{n}}_{i}, y_{i}^{*(M+1)}\right)$ given by

$$
\begin{aligned}
& \boldsymbol{y}_{1}^{*}=\frac{1}{2}\left(\theta \boldsymbol{x}^{*}-\boldsymbol{w}^{*}\right) \\
& \boldsymbol{y}_{2}^{*}=-\frac{1}{2}\left(\theta \boldsymbol{x}^{*}+\boldsymbol{w}^{*}\right),
\end{aligned}
$$

belong to the pencils containing the dual lines $L^{*}=x^{*} \cap w^{*}$. Equations (21)-(22) imply that $\boldsymbol{y}_{i}^{*}$ satisfy the system (12), therefore $y_{i}^{*}$ are dual focal lattices of the dual congruence $L^{*}$ conjugate to the dual Koenigs lattice $x^{*}$.

Due to equations (19)-(20) the normal vectors $\overrightarrow{\boldsymbol{n}}_{i}, i=1$, 2, of the dual focal lattices $\boldsymbol{y}_{i}^{*}$, given by equations (25)-(26), satisfy conditions

$$
\overrightarrow{\boldsymbol{n}}_{2} \cdot \overrightarrow{\boldsymbol{n}}_{2}=\theta \theta_{(1)}\left(U_{1}+\lambda\right), \quad \overrightarrow{\boldsymbol{n}}_{1} \cdot \overrightarrow{\boldsymbol{n}}_{1}=\theta \theta_{(2)}\left(U_{2}-\lambda\right) .
$$


Then the vectors $\overrightarrow{\boldsymbol{n}}_{1} / \sqrt{\theta \theta_{(2)}}$ and $\overrightarrow{\boldsymbol{n}}_{1(1)} / \sqrt{\theta_{(1)} \theta_{(12)}}$ are of equal length, and the hyperplanes bisecting $y_{1}^{*}$ and $y_{1(1)}^{*}$ have the normal vectors

$$
\overrightarrow{\boldsymbol{b}}_{1 \pm}=\frac{\overrightarrow{\boldsymbol{n}}_{1}}{\sqrt{\theta \theta_{1(1)}}} \pm \frac{\overrightarrow{\boldsymbol{n}}_{1(1)}}{\sqrt{\theta_{(1)} \theta_{(12)}}} .
$$

Similarly, the hyperplanes bisecting $y_{2}^{*}$ and $y_{2(2)}^{*}$ have the normal vectors

$$
\overrightarrow{\boldsymbol{b}}_{2 \mp}=\frac{\overrightarrow{\boldsymbol{n}}_{2}}{\sqrt{\theta \theta_{2(2)}}} \pm \frac{\overrightarrow{\boldsymbol{n}}_{2(2)}}{\sqrt{\theta_{(2)} \theta_{(12)}}} .
$$

Equations (18), (23) and (24) imply that

$$
\overrightarrow{\boldsymbol{b}}_{1-} \cdot \overrightarrow{\boldsymbol{b}}_{2+}=\overrightarrow{\boldsymbol{b}}_{1+} \cdot \overrightarrow{\boldsymbol{b}}_{2-}=0,
$$

which shows that the dual congruence $L^{*}$ is normal.

COROLlARY 14. Once the normal dual congruence $L^{*}$ is found then the homogeneous coordinates of the corresponding new dual Bianchi lattice $x^{* \prime}$ are given in terms of $\boldsymbol{w}^{*}$ by

$$
\boldsymbol{x}^{*}=\frac{1}{2}\left[\left(\frac{1}{\theta_{(1)}}-\frac{1}{\theta_{(2)}}\right) \theta \boldsymbol{x}^{*}+\left(\frac{1}{\theta_{(1)}}+\frac{1}{\theta_{(2)}}\right) \boldsymbol{w}^{*}\right] .
$$

Following $[\mathbf{1 7}, \mathbf{1 6}]$ one can formulate the transition from the normal vectors $\overrightarrow{\boldsymbol{n}}$ of the dual Bianchi lattice $x^{*}$ to the normal vectors $\overrightarrow{\boldsymbol{n}}^{\prime}$ of the new dual Bianchi lattice $x^{* \prime}$ as follows.

LEMMA 15. Given $\overrightarrow{\boldsymbol{n}}: \mathbb{Z}^{2} \rightarrow \mathbb{E}^{M}$ satisfying the discrete Koenigs equation (4) with the constraint (18), define $\overrightarrow{\boldsymbol{\eta}}_{0}=F \overrightarrow{\boldsymbol{n}}$ and supplement it by unit vectors $\overrightarrow{\boldsymbol{\eta}}_{A}, A=1, \ldots, M-1$ to an orthogonal basis. Denote by $p_{A}^{B}, q_{A}^{B}, A, B=0,1, \ldots, M-1$, the functions given by the unique decompositions

$$
\overrightarrow{\boldsymbol{\eta}}_{A}=\sum_{B=0}^{M-1} p_{A}^{B} \overrightarrow{\boldsymbol{\eta}}_{B(1)}, \quad \overrightarrow{\boldsymbol{\eta}}_{A}=\sum_{B=0}^{M-1} q_{A}^{B} \overrightarrow{\boldsymbol{\eta}}_{B(2)},
$$

and let $R=U_{1}+U_{2}, a=U_{1}+\lambda, b=U_{2}-\lambda$. Then:

(i) the linear system

$$
\Theta_{(i)}=M_{i} \Theta, \quad i=1,2,
$$

where $\Theta=\left(\theta, \theta^{\prime}, \theta^{\prime \prime}, y^{1}, \ldots, y^{M-1}\right)^{T}$, and

$$
M_{1}=\left(\begin{array}{cccccc}
0 & 1 & 0 & 0 & \cdots & 0 \\
\frac{R_{(1)} \frac{p_{0}^{0}}{F_{(1)}}-b}{-1} & \frac{b F^{*}-R_{(1)}\left(\frac{R+b}{R} p_{0}^{0}-\frac{1}{F_{(1)}^{*}}\right)}{a_{(1)}} & \frac{b}{a_{(1)}}\left(F^{*}-\frac{R_{(1)}}{R} p_{0}^{0}\right) & \frac{R_{(1)}}{a_{(1)}} p_{1}^{0} & \cdots & \frac{R_{(1)}}{a_{(1)}} p_{M-1}^{0} \\
\frac{F^{*}}{F^{*}} & -\frac{R+b}{R} p_{0}^{1} & F^{*} & 0 & \cdots & 0 \\
\vdots & \vdots & -\frac{b}{R} p_{0}^{1} & p_{1}^{1} & \cdots & p_{M-1}^{1} \\
\frac{p_{0}^{M-1}}{F^{*}} & -\frac{R+b}{R} p_{0}^{M-1} & \vdots & \vdots & \ddots & \vdots \\
& & -\frac{b}{R} p_{0}^{M-1} & p_{1}^{M-1} & \cdots & p_{M-1}^{M-1}
\end{array}\right),
$$




$$
M_{2}=\left(\begin{array}{cccccc}
0 & 0 & 1 & 0 & \cdots & 0 \\
-1 & F^{*} & F^{*} & 0 & \cdots & 0 \\
\frac{R_{(2)} \frac{q_{0}^{0}}{F_{(2)}}-a}{b_{(2)}} & \frac{a}{b_{(2)}}\left(F^{*}-\frac{R_{(2)}}{R} q_{0}^{0}\right) & \frac{a F^{*}-R_{(2)}\left(\frac{R+a}{R} q_{0}^{0}-\frac{1}{F_{(2)}^{*}}\right)}{b_{(2)}} & -\frac{R_{(2)}}{b_{(2)}} q_{1}^{0} & \cdots & -\frac{R_{(2)}}{b_{(2)}} q_{M-1}^{0} \\
-\frac{q_{0}^{1}}{F^{*}} & \frac{a}{R} q_{0}^{1} & \frac{a+R}{R} q_{0}^{1} & q_{1}^{1} & \cdots & q_{M-1}^{1} \\
\vdots & \vdots & \vdots & \vdots & \ddots & \vdots \\
-\frac{q_{0}^{M-1}}{F^{*}} & \frac{a}{R} q_{0}^{M-1} & \frac{a+R}{R} q_{0}^{M-1} & q_{1}^{M-1} & \cdots & q_{M-1}^{M-1}
\end{array}\right),
$$

is compatible;

(ii) the function

$$
I=\left(y^{1}\right)^{2}+\cdots+\left(y^{M-1}\right)^{2}+\frac{R}{F^{*}} \theta^{2}+\frac{F^{*}}{R}\left(b \theta^{\prime \prime}-a \theta^{\prime}\right)^{2}-2 \theta\left(a \theta^{\prime}+b \theta^{\prime \prime}\right),
$$

is a first integral of the system;

(iii) the function $\overrightarrow{\boldsymbol{\omega}}=\sum_{A=0}^{M-1} y^{A} \overrightarrow{\boldsymbol{\eta}}_{A}$ with

$$
y^{0}=\frac{a \theta^{\prime}-b \theta^{\prime \prime}}{R},
$$

satisfies the linear system (21)-(22) with $\overrightarrow{\boldsymbol{n}}$ in place of $\boldsymbol{x}^{*}$.

COROLlaRY 16. The solution $\theta$ of the linear system (27) is a solution of the discrete Moutard equation (6), while $\theta^{\prime}=\theta_{(1)}$ and $\theta^{\prime \prime}=\theta_{(2)}$.

Proposition 17. Given $\overrightarrow{\boldsymbol{n}}: \mathbb{Z}^{2} \rightarrow \mathbb{E}^{M}$ satisfying the discrete Bianchi system (4), (18), let $\theta$ and $\vec{\omega}$ be obtained from the solution of the linear system (27) subjected to the admissible constraint $I=0$. Then $\overrightarrow{\boldsymbol{\omega}}$ satisfies equations (23)-(24) and $\overrightarrow{\boldsymbol{n}}^{\prime}$, given by equation (14) with $\overrightarrow{\boldsymbol{n}}$ and $\overrightarrow{\boldsymbol{\omega}}$ in place of $\boldsymbol{x}^{*}$ and $\boldsymbol{w}^{*}$, is a new solution of the system.

ACKNOWLEDGMENTS. The author would like to thank organizers of the meeting ISLAND 2 for support during the conference. The paper was supported in part by the University of Warmia and Mazury grant 522-1307-0201, and by the KBN grant 2P03B12622.

\section{REFERENCES}

1. A. A. Akhmetshin, I. M. Krichever and Y. S. Volvovski, Discrete analogs of the Darboux-Egoroff metrics, Proc. Steklov Inst. Math. 225 (1999), 16-39.

2. L. Bianchi, Sulle varietà a tre dimensioni deformabili entro lo spazio Euclideo a quattro dimensioni, Mem. Soc. It. delle Sc. 13 (1905), 261-.

3. L. Bianchi, Lezioni di geometria differenziale (Zanichelli, Bologna, 1924).

4. A. Bobenko, Discrete conformal maps and surfaces, in Symmetries and Integrability of Difference Equations II (P. Clarkson and F. Nijhoff, eds.) (Cambridge University Press, 1999), 97-108.

5. A. Bobenko and U. Pinkall, Discrete isothermic surfaces, J. Reine Angew. Math. $\mathbf{4 7 5}$ (1996), 187-208.

6. A. Bobenko and U. Pinkall, Discretization of surfaces and integrable systems, in Discrete Integrable Geometry and Physics (A. Bobenko and R. Seiler, eds.) (Clarendon Press, Oxford, 1999), 3-58.

7. J. Cieśliński, A. Doliwa and P. M. Santini, The integrable discrete analogues of orthogonal coordinate systems are multidimensional circular lattices, Phys. Lett. A 235 (1997), 480488 . 

192.

8. A. Doliwa, Geometric discretisation of the Toda system, Phys. Lett. A 234 (1997), 187-

9. A. Doliwa, Quadratic reductions of quadrilateral lattices, J. Geom. Phys. 30 (1999), $169-186$.

10. A. Doliwa, Discrete asymptotic nets and W-congruences in Plücker line geometry, J. Geom. Phys. 39 (2001), 9-29.

11. A. Doliwa, Integrable multidimensional discrete geometry: Quadrilateral lattices, their transformations and reductions, Integrable Hierarchies and Modern Physical Theories (H. Aratyn and A. S. Sorin, eds.), Kluwer, Dordrecht, 2001, pp. 355-389.

12. A. Doliwa, The Ribaucour congruences of spheres within Lie sphere geometry, Bäcklund and Darboux Transformations: The Geometry of Soliton Theory, Halifax, 1999 (C. Rogers and P. Winternitz, eds.), CMR Proceedings and Lecture Notes, vol. 29, AMS, Providence, 2001, pp. 159-166.

13. A. Doliwa, Geometric discretization of the Koenigs nets, J. Math. Phys. 44 (2003), 2234-2249.

14. A. Doliwa, S. V. Manakov and P. M. Santini, $\bar{\partial}$-reductions of the multidimensional quadrilateral lattice: the multidimensional circular lattice, Comm. Math. Phys. 196 (1998), 1-18.

15. A. Doliwa, M. Mañas and L. Martínez Alonso, Generating quadrilateral and circular lattices in KP theory, Phys. Lett. A 262 (1999), 330-343.

16. A. Doliwa, M. Nieszporski and P. M. Santini, Geometric discretization of the Bianchi system, J. Geom. Phys. 52 (2004), 217-240.

17. A. Doliwa, M. Nieszporski and P. M. Santini, Asymptotic lattices and their integrable reductions: I. the Bianchi-Ernst and the Fubini-Ragazzi lattices, J. Phys. A: Math. Gen. 34 (2001), 10423-10439.

18. A. Doliwa and P. M. Santini, Integrable dynamics of a discrete curve and the AblowitzLadik hierarchy, J. Math. Phys. 36 (1995), 1259-1273.

19. A. Doliwa and P. M. Santini, Multidimensional quadrilateral lattices are integrable, Phys. Lett. A 233 (1997), 365-372.

20. A. Doliwa and P. M. Santini, The symmetric, D-invariant and Egorov reductions of the quadrilateral lattice, J. Geom. Phys. 36 (2000), 60-102.

21. A. Doliwa, P. M. Santini and M. Mañas, Transformations of quadrilateral lattices, J. Math. Phys. 41 (2000), 944-990.

22. L. P. Eisenhart, A treatise on the differential geometry of curves and surfaces (Ginn and Company, Boston, 1909).

23. L. P. Eisenhart, Transformations of surfaces (University Press, Princeton, 1923).

24. B. G. Konopelchenko and W. K. Schief, Three-dimensional integrable lattices in Euclidean spaces: Conjugacy and orthogonality, Proc. Roy. Soc. London A 454 (1998), 30753104.

25. Q. P. Liu and M. Mañas, Superposition formulae for the discrete Ribaucour transformations of circular lattices, Phys. Lett. A 249 (1998), 424-430.

26. Th. F. Moutard, Sur la construction des équations de la forme $\frac{1}{z} \frac{\partial^{2} z}{\partial x \partial y}=\lambda(x, y)$, qui admettenent une intégrale générale explicite, J. Éc. Pol. 45 (1878), 1-11.

27. M. Nieszporski, The multicomponent Ernst equation and the Moutard transformation, Phys. Lett. A 272 (2000), 74-79.

28. M. Nieszporski, On discretization of asymptotic nets, J. Geom. Phys. 40 (2001), 259-276.

29. M. Nieszporski, A Laplace ladder of discrete Laplace equations, Theor. Math. Phys. 133 (2002), 1576-1584.

30. M. Nieszporski, A. Doliwa and P. M. Santini, The integrable discretization of the Bianchi-Ernst system, nlin.SI/0104065.

31. J. J. C. Nimmo and W. K. Schief, Superposition principles associated with the Moutard transformation. An integrable discretisation of a $(2+1)$-dimensional sine-Gordon system, Proc. Roy. Soc. London A 453 (1997), 255-279.

32. W. K. Schief, Isothermic surfaces in spaces of arbitrary dimension: integrability, discretization and Bäcklund transformations - A discrete Calapso equation, Stud. Appl. Math. 106 (2001), 85-137.

33. W. K. Schief, On the integrability of infinitesimal and finite deformations of polyhedral surfaces, talk presented at the conference "Integrable Systems: Linear and Nonlinear Dynamics 2", Isle of Arran, June 2003. 
